\title{
Pixel size limit in holographic memories
}

\author{
Wenhai Liu and Demetri Psaltis \\ Department of Electrical Engineering, California Institute of Technology, Mail Stop 136-93, Pasadena, California 91125
}

Received June 28, 1999

\begin{abstract}
The bandwidth of holographic recording in $\mathrm{LiNbO}_{3}$ ( $\mathrm{Fe}$ doped) in the $90^{\circ}$ geometry is studied theoretically and experimentally. The wide holographic bandwidth of $\mathrm{LiNbO}_{3}$ makes it possible to record submicrometer pixels and reconstruct them by phase conjugation in a holographic memory system. This approach reduces the system cost and increases the system storage density. We demonstrate the recording and the phase-conjugate reconstruction of various pixel sizes down to $1 \mu \mathrm{m} \times 1 \mu \mathrm{m}$. The signal-noise ratio and the bit-error rate are examined. (c) 1999 Optical Society of America

OCIS codes: $170.4500,170.2150,170.2680,170.4580,110.4500,170.0110$.
\end{abstract}

In this Letter we demonstrate that it is possible to record holographically $1-\mu \mathrm{m}$ pixels in $\mathrm{LiNbO}_{3}(\mathrm{Fe}$ doped) in the $90^{\circ}$ geometry by use of the compact phaseconjugate readout architecture. ${ }^{1}$ This high density is a consequence of the wide recording bandwidth of the photorefractive medium and the phase-conjugate readout method. Figure 1 shows one possible implementation of the angle-multiplexed phase-conjugate holographic memory module ${ }^{1}$ that consists of a spatial light modulator, a pixel-matched detector array, a photorefractive crystal, and multiplexing optical elements. Information is recorded by the interference between the spatial light modulator-encoded signal beam and the plane-wave reference. The stored information is retrieved by illumination of the crystal with a plane-wave reference beam that is propagating in a direction opposite the recording reference beam. The counterpropagating reference is generated by reflection from the mirror on the opposite side of the crystal. This reflection results in the phase-conjugate readout of the stored hologram and the formation of a real image on the pixel-matched detector array. Multiple holograms can be stored in the same crystal by use of angle multiplexing. As shown in Fig. 1, a separate laser source is used for each hologram; lens $\mathrm{L}$ converts the position of the laser to the angle of incidence on the crystal. The signal beam enters the crystal at different angles for various spatialfrequency components with corresponding polarization directions. During recording, these spatial-frequency components generate interference grating vectors of different direction, period, and modulation depth with the reference beam. These interference patterns then form different space-charge-field gratings. The dependence of the grating strength on its direction and spatial frequency leads to angle-dependent diffraction efficiencies that correspond to various signal spatialfrequency components. ${ }^{2}$ This holographic bandwidth of the medium determines the maximum spatial frequency, or the minimum feature size that can be recorded and reconstructed in the phase-conjugate geometry.

We modeled the angle-dependent recording efficiency in the $90^{\circ}$ geometry for $\mathrm{LiNbO}_{3}(\mathrm{Fe})$ as shown in Fig. 1. The crystal is $45^{\circ}$ cut, with the $c$ axis along $(1,-1,0)$. The signal beam inside the crystal consists of wave vectors $\left(k_{x}, k_{y}, k_{z}\right)$ with polarization $\mathbf{P}_{S}$, where $k_{x}{ }^{2}+k_{y}{ }^{2}+k_{z}{ }^{2}=k_{0}{ }^{2}=(2 \pi n / \lambda)^{2}$ and $n$ is the refractive index:

$$
\begin{aligned}
\mathbf{P}_{S}= & {\left[-\frac{k_{x} k_{z}}{k_{0}\left(k_{x}^{2}+k_{y}^{2}\right)^{1 / 2}},\right.} \\
& \left.-\frac{k_{y} k_{z}}{k_{0}\left(k_{x}^{2}+k_{y}\right)^{1 / 2}}, \frac{\left(k_{x}^{2}+k_{y}{ }^{2}\right)^{1 / 2}}{k_{0}}\right] .
\end{aligned}
$$

The reference beam enters the crystal with wave vector $\left(0, k_{0}, 0\right)$ and polarization $\mathbf{P}_{R}=(0,0,1)$.

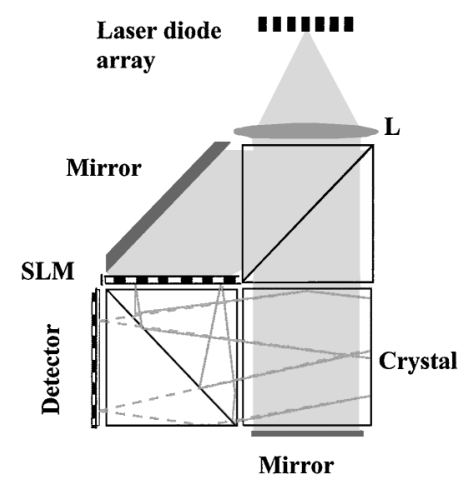

(a)

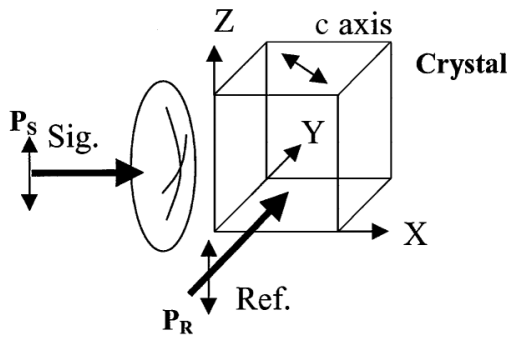

(b)

Fig. 1. (a) Compact holographic memory module with phase-conjugate readout. (b) $90^{\circ}$ recording geometry. SLM, spatial light modulator; Sig., signal; Ref., reference. 


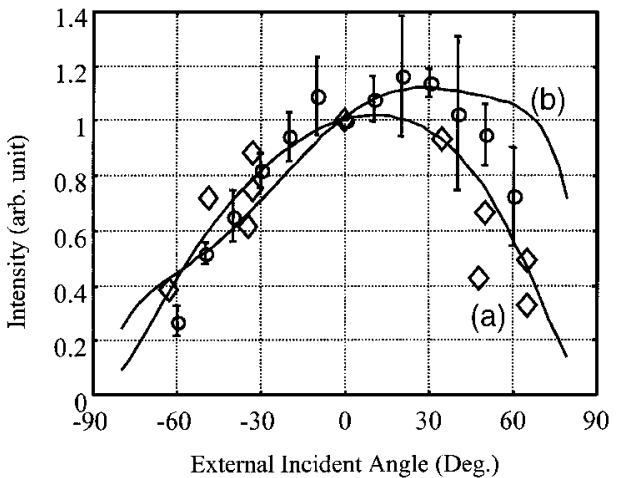

Fig. 2. Curve (a), experimental data (diamonds) and the theoretical calculation of holographic efficiency in the signal reference plane. Curve (b), experimental data (circles) and the theoretical calculation of the holographic efficiency out of the signal reference plane.

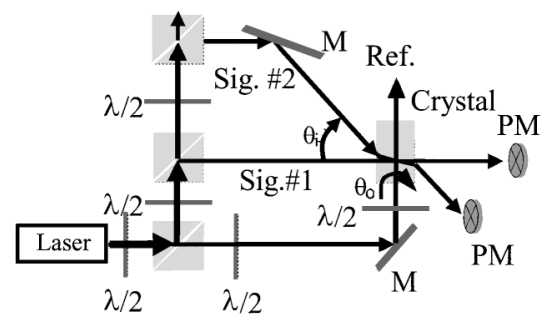

Fig. 3. Experimental setup for holographic bandwidth measurement: M's, mirrors; Sig.\#1, Sig.\#2, signal beams; Ref., reference beam; PM's, powermeters; $\lambda / 2$ 's, halfwavelength plates. For in-plane measurement, Sig.\#2 rotates by $\theta_{i}$ and is adjusted to have the same intensity as the normally incident Sig.\#1 inside the crystal by consideration of the Fresnel reflection loss. The diffraction efficiency of Sig.\#2 is then measured relative to the Sig.\#1 diffraction efficiency. For out-of-plane measurement, only the Sig.\#1 is used, and the crystal and the reference polarization direction rotate by $\theta_{o}$ about the reference-beam direction.

The grating vector is $G \mathbf{g}=\left(k_{x}, k_{y}-k_{0}, k_{z}\right)$. Both the magnitude $G$ and the direction $\mathbf{g}$ are functions of $k_{y}$ and $k_{z}$. Given the parameters of the material, ${ }^{3}$ such as total Fe-dopant density, initial $\mathrm{Fe}^{3+}$ concentration, acceptor density, photovoltaic parameter, photoexcitation cross section, carrier combination rate, and carrier mobility, we can calculate the spatial-charge-field grating $E_{g} \mathbf{g}$ by solving the Kukhtarev equations. ${ }^{4}$ The effective phase grating as a function of $k_{y}$ and $k_{z}$ is thus given by $\Delta \epsilon=\mathbf{P}_{S} \cdot(\overline{\overline{\mathbf{r}}} \cdot \mathbf{g}) \cdot \mathbf{P}_{R} E_{g}$, where $\overline{\overline{\mathbf{r}}}$ is the electrooptic tensor. In addition to the angle-dependent phase grating, the reflection losses at the crystal-air interface also depend on the incident angle and the polarization, and they are included in our model. We examine two special cases in detail. First, all the signal-beam components are assumed to be in the $x-y$ plane $\left(k_{z}=0\right)$ and to have the same polarization along $(0,0,1)$. The grating vector has different direction and magnitude as a function of $k_{y}$. The modulation depth also changes owing to the angle-dependent reflection losses. In the second case we consider the wave vectors of all signal components and their polarization tilt in the $x-y$ plane $\left(k_{y}=0\right)$.
Combining the effects of both angle-dependent phase grating and reflection loss without antireflection coating, we calculated the holographic recording and reconstruction efficiencies as functions of the signal external incident angle corresponding to spatial frequencies $k_{y}$ and $k_{z}$ for the two cases described above, as shown in Fig. 2. The figure shows that the wide holographic bandwidth of $\mathrm{LiNbO}_{3}(\mathrm{Fe})$ is capable of recording submicrometer patterns with a wavelength of $0.5 \mu \mathrm{m}$. The material parameters used in our model are the same as those described in Ref. 3. We performed an experiment to confirm the theoretical prediction illustrated in Fig. 2, using the experimental apparatus shown in Fig. 3. This setup allowed us to record and measure gratings in a $90^{\circ}$-geometry $\mathrm{LiNbO}_{3}$ crystal in the range of $\pm 70^{\circ}$ external angle of the signal beam with regard to the crystal normal. Measurements were performed for both the in-plane and the out-of-plane geometry, and the results are plotted in Fig. 2 as well. Good agreement between theory and experiment is obtained.

A resolution mask with pixel sizes varying from $2 \mu \mathrm{m} \times 2 \mu \mathrm{m}$ down to $0.2 \mu \mathrm{m} \times 0.2 \mu \mathrm{m}$ was used to record holograms in $\mathrm{LiNbO}_{3}$ in the phase-conjugate structure shown in Fig. 1. The direct image of the pattern, magnified by a Nikon objective lens (N.A., 0.65), is shown in Fig. 4(a). The phase-conjugate reconstruction magnified by the same lens is shown in Fig. 4(b), with no degradation of the submicrometer patterns from the direct image. This not only confirms the holographic bandwidth for recording submicrometer features but also indicates that the

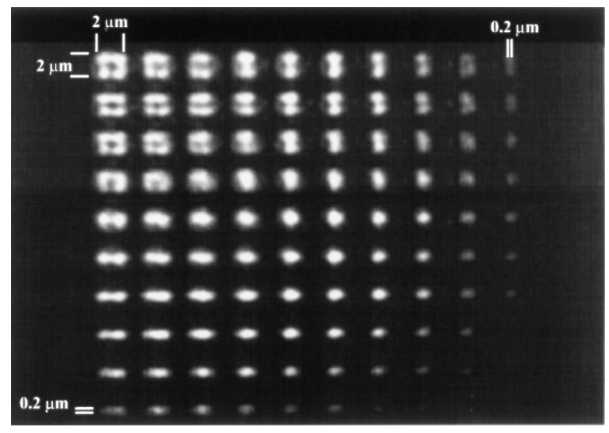

(a)

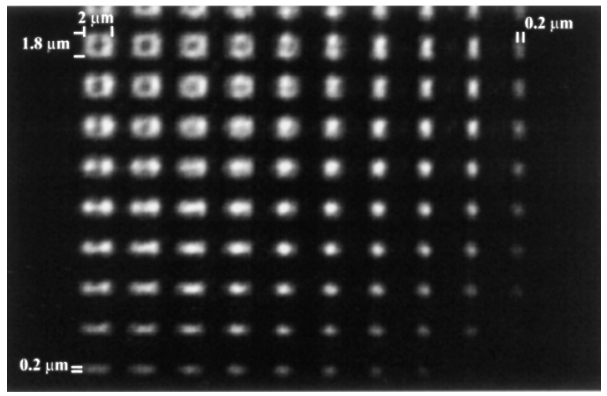

(b)

Fig. 4. (a) Direct image of a resolution photo mask with pixels from $2 \mu \mathrm{m} \times 2 \mu \mathrm{m}$ down to $0.2 \mu \mathrm{m} \times 0.2 \mu \mathrm{m}$. (b) Holographic phase-conjugate reconstruction of the photo mask. Both images were magnified by a Nikon objective lens with a N.A. of 0.65 . 


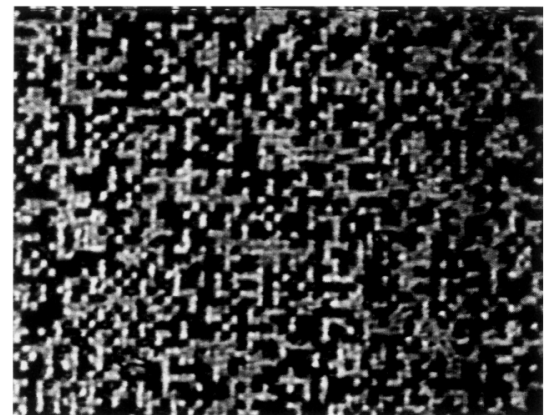

(a)

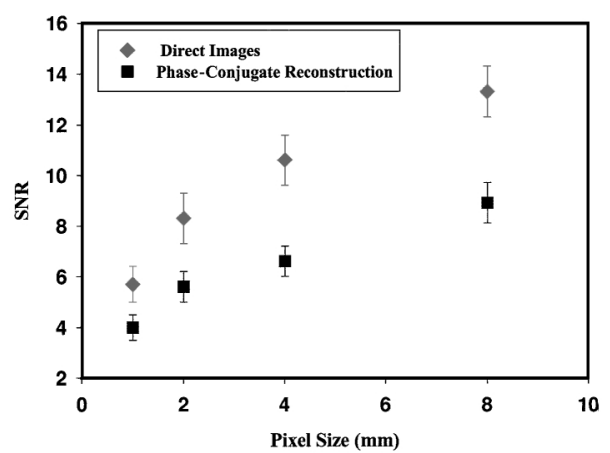

(b)

Fig. 5. (a) Phase-conjugate reconstruction of $1 \mu \mathrm{m} \times$ $1 \mu \mathrm{m}$ random data mask hologram. (b) Signal-noise ratio (SNR) of the direct images and the holographic phaseconjugate reconstruction of random binary data with pixel sizes from $8 \mu \mathrm{m} \times 8 \mu \mathrm{m}$ down to $1 \mu \mathrm{m} \times 1 \mu \mathrm{m}$.

resolution of the phase-conjugate reconstruction is comparable with or better than the resolution of the imaging lens (N.A., 0.65).

In addition, the phase-conjugate readout provides a mechanism to record and reconstruct all the signaldiffraction components that are reflected by the walls in the recording medium. We observed that the quality of the phase-conjugate reconstruction was not de- graded even at the edge of the crystal, even though the signal beam fully illuminated the crystal aperture. This result means that the high-spatial-frequency signal components near the edge of the entrance aperture are reflected by the sidewall, recorded, and faithfully reconstructed.

Finally, we recorded holograms of random binary data masks with pixel sizes of $8 \mu \mathrm{m} \times 8 \mu \mathrm{m}$ down to $1 \mu \mathrm{m} \times 1 \mu \mathrm{m}$ in the $90^{\circ}$ geometry. The phaseconjugate reconstruction signal-noise ratio was measured and is shown in Fig. 5, in which the signalnoise ratio is defined as the difference of the ON and OFF pixels divided by the square root of the sum of the deviations. The bit-error rate for the $1 \mu \mathrm{m} \times 1 \mu \mathrm{m}$ holograms is estimated to be $7 \times 10^{-5}$, assuming a Gaussian distribution for the ON and OFF pixels.

In conclusion, we have studied the holographic bandwidth and demonstrated the submicrometer limit of the pixel size in a holographic memory system. It is crucial to record and reconstruct small pixels for a holographic memory system to reduce the system cost and make it commercially competitive. However, we must also develop spatial light modulators and detector arrays with $1-\mu \mathrm{m}$ pixels to match the holographic bandwidth.

This work was supported by the Jet Propulsion Laboratory as part of the Hybrid Technology Multithreaded Architecture and by Rome Labs. We thank Ernest Chuang and George Barbastathis for helpful discussions. D. Psaltis's e-mail address is psaltis@caltech.edu.

\section{References}

1. J-J. P. Drolet, E. Chuang, G. Barbastathis, and D. Psaltis, Opt. Lett. 22, 552 (1997).

2. H. Zhou, F. Zhao, and F. Yu, Appl. Opt. 34, 1303 (1995).

3. G. W. Burr and D. Psaltis, Opt. Lett. 21, 893 (1996).

4. P. Yeh, Introduction to Photorefractive Nonlinear Optics (Wiley, New York, 1993). 